\title{
Hospital case-volume is associated with case-fatality after aneurysmal subarachnoid hemorrhage
}

\section{Imperial Coll Longon}

2019-04

Imperial Coll Longon, Lindgren , A , Burt , S , Turner , E B , Meretoja , A , Lee , J-M , Hemmen, T M , Alberts, M , Lemmens, R, Vergouwen, M D I \& Rinkel , G J E 2019 , ' Hospital case-volume is associated with case-fatality after aneurysmal subarachnoid hemorrhage ' , International Journal of Stroke , vol. 14 , no. 3 , pp. 282-289 . https://doi.org/10.1177/1747493018790

http://hdl.handle.net/10138/314534

https://doi.org/10.1177/1747493018790073

publishedVersion

Downloaded from Helda, University of Helsinki institutional repository.

This is an electronic reprint of the original article.

This reprint may differ from the original in pagination and typographic detail.

Please cite the original version. 


\section{Hospital case-volume is associated with case-fatality after aneurysmal subarachnoid hemorrhage}

International Journal of Stroke 2019, Vol. 14(3) 282-289 (C) 2018 World Stroke Organization Article reuse guidelines: sagepub.com/journals-permissions DOI: $10.1177 / 1747493018790073$ journals.sagepub.com/home/wso @SAGE

\author{
Antti Lindgren ${ }^{1,2}{ }^{(0}$, Sarah Burt ${ }^{3}$, Ellie Bragan Turner ${ }^{3}$, \\ Atte Meretoja ${ }^{4,5}$, Jin-Moo Lee ${ }^{6}$, Thomas M Hemmen ${ }^{7}$, \\ Mark Alberts ${ }^{8}$, Robin Lemmens ${ }^{9,10,11}$, Mervyn DI Vergouwen' (1) \\ and Gabriel JE Rinkel'; on behalf of the Stroke GOAL Group, \\ Dr Foster Global Comparators Project, Dr Foster Ltd in \\ association with the Dr Foster Unit at Imperial College London
}

\begin{abstract}
Background: Inverse association between hospital case-volume and case-fatality has been observed for various nonsurgical interventions and surgical procedures.

Aims: To study the impact of hospital case-volume on outcome after aneurysmal subarachnoid hemorrhage (aSAH).

Methods: We included aSAH patients who underwent aneurysm coiling or clipping from tertiary care medical centers across three continents using the Dr Foster Stroke GOAL database 2007-2014. Hospitals were categorized by annual case-volume (low volume: <4I/year; intermediate: 4I-70/year; high: >70/year). Primary outcome was I4-day in-hospital case-fatality. We calculated proportions, and used multiple logistic regression to adjust for age, sex, differences in comorbidity or disease severity, aneurysm treatment modality, and hospital.
\end{abstract}

Results: We included 8525 patients (2363 treated in low volume hospitals, 3563 treated in intermediate volume hospitals, and 2599 in high-volume hospitals). Crude 14-day case-fatality for hospitals with low case-volume was 10.4\% (95\% confidence interval $(\mathrm{Cl}) 9.2-11.7 \%)$, for intermediate volume 7.0\% (95\% Cl 6.2-7.9\%; adjusted odds ratio (OR) $0.63(95 \% \mathrm{Cl} 0.47-0.85))$ and for high volume $5.4 \%$ (95\% Cl 4.6-6.3\%; adjusted OR 0.50 (95\% Cl 0.33-0.74)). In patients with clipped aneurysms, adjusted OR for I4-day case-fatality was 0.46 ( $95 \% \mathrm{Cl} 0.30-0.7 \mathrm{I})$ for hospitals with intermediate case-volume and $0.42(95 \% \mathrm{Cl} 0.25-0.72)$ with high case-volume. In patients with coiled aneurysms, adjusted $\mathrm{OR}$ was $0.77(95 \% \mathrm{Cl} 0.55-1.07)$ for hospitals with intermediate case-volume and $0.56(95 \% \mathrm{Cl} 0.36-0.87)$ with high case-volume.

Conclusions: Even within a subset of large, tertiary care centers, intermediate and high hospital case-volume is associated with lower case-fatality after aSAH regardless of treatment modality, supporting centralization to higher volume centers.

\title{
Keywords
}

Subarachnoid hemorrhage, intracranial aneurysm, outcome, hospital volume, epidemiology, stroke

Received: 31 January 2018; accepted: 8 May 2018

\footnotetext{
'Department of Neurology and Neurosurgery, University Medical Center Utrecht, Utrecht, The Netherlands

${ }^{2}$ Department of Neurosurgery, NeuroCenter, Kuopio University Hospital, Kuopio, Finland

${ }^{3}$ Dr Foster Ltd, London, UK

${ }^{4}$ Department of Neurology, Helsinki University Hospital, Helsinki, Finland

${ }^{5}$ Department of Medicine at the Royal Melbourne Hospital, University of Melbourne, Parkville, Australia

${ }^{6}$ Department of Neurology, and the Hope Center for Neurological disorders, Washington University School of Medicine, St. Louis, MO, USA ${ }^{7}$ Department of Neurosciences, University of California, San Diego, CA, USA
}

\footnotetext{
${ }^{8}$ Department of Neurology, Hartford Hospital, Hartford, CT, USA

${ }^{9} \mathrm{KU}$ Leuven - University of Leuven, Department of Neurosciences, Experimental Neurology, Leuven Institute for Neuroscience and Disease (LIND), Leuven, Belgium

${ }^{10}$ VIB, Center for Brain \& Disease Research, Laboratory of Neurobiology, Leuven, Belgium

"Department of Neurology, University Hospitals Leuven, Leuven, Belgium

Corresponding author:

Antti Lindgren, Department of Neurosurgery, NeuroCenter, Kuopio University Hospital, P.O. Box 1777, Kuopio 702II, Finland.

Email: antti.lindgren@kuh.fi
} 


\section{Introduction}

The relationship between hospital volumes and treatment outcomes has been a timely topic for the past decade, partly due to pressure towards centralization of specialized health care services. An inverse association between hospital case-volume and case-fatality has previously been observed for various nonsurgical interventions and surgical procedures, ${ }^{1}$ including treatment of acute ischemic stroke ${ }^{2}$ and unruptured intracranial aneurysms. ${ }^{3,4}$ Some previous studies from the United States and United Kingdom also suggested that aneurysmal subarachnoid hemorrhage (aSAH) patients treated at high-volume centers have better outcomes, ${ }^{3,5-12}$ but other studies have failed to show this association. ${ }^{13-17}$ The contradicting results might be explained by variation in the definitions of a "highvolume center", with lower border for high volume being in some studies no more than 15 or 20 patients per year ${ }^{5,9,11}$ and, consequently, large variation in numbers of patients in the category "high volume". In other studies, lower borders for high volume were as high as 100 per year. $^{3}$ Other explanations for the different results between the different studies include failure to adjust for aSAH severity, variable inclusion of nonaneurysmal subarachnoid hemorrhages, and different outcome variables.

Most of the previous studies were performed using administrative datasets from hospitals in one or more states from the United States, and only one previous study was international, ${ }^{13}$ but restricted to Scandinavian countries. Thus, the generalizability of these results to other health care systems has been questioned.

\section{Hypothesis}

We studied the effect of hospital volume on casefatality in aSAH patients after treatment by coiling or clipping in a large, international administrative dataset collected from tertiary care centers in three continents with the hypothesis that high hospital aSAH case-volume is associated with lower case-fatality after aSAH.

\section{Methods}

\section{Study design and patients}

For this nonrandomized comparative study, we used the Dr Foster Global Comparators International dataset. This international, multicenter dataset consists of anonymized in-hospital administrative medical records data, provided individually by member hospitals from three continents, and is designed for research and quality improvement utilizing benchmarking data from multiple international medical centers. The process of reconciling the differing diagnostic coding systems across countries and integration of the medical data into a uniform dataset has been described in a prior report. ${ }^{18}$

For this study, we used data from 10 hospitals in Europe (one in Belgium, five in United Kingdom, three in the Netherlands, and one in Norway), eight in the United States, and four in Australia. All hospitals are tertiary care hospitals. From these hospitals we retrieved data of patients with aSAH (ICD 9 codes 430 and ICD 10 codes I60.0-9), discharged between 2007 and 2014.

For the analyses, we used only those patients in whom the aneurysm had been occluded by means of neurosurgical clipping or endovascular coiling. Thus, patients without aneurysm treatment were excluded from the analyses. We extracted data on age, sex, type of aneurysm occlusion (clipping or coiling), comorbidity index, length of stay, and in-hospital case-fatality.

\section{Statistical analysis}

The primary outcome measure was in-hospital casefatality at 14 days. Since the administrative dataset has no information on clinical condition on admission, we identified a list of conditions relevant for comorbidity or being proxy for disease severity for risk adjustment (Table 1).

We calculated crude 14-day case-fatality rates with corresponding 95\% confidence intervals (CI). Median annual hospital volumes were calculated and hospitals were categorized into three groups according to their numbers of aSAH patients treated per year (low volume: 0 to 40 aSAH patients/year; intermediate volume: 41 to 70 aSAH patients/year; high volume: more than 70 aSAH patients/year). Cut-offs were chosen based on the number of patients per hospital only, to balance the numbers of patients. Crude casefatality rates were plotted against hospital volumes as scatter plots for all patients combined and for patients with aneurysm clipping or coiling separately. The scatter plots are shown with logarithmic fit lines.

Two logistic regression models were used. In the first model, case-fatality at 14 days was used as the outcome and median annual hospital volume was input as a categorical variable with age, sex, type of treatment, and separate comorbidity types (Table 1) as other variables. Length of stay did not show a correlation with 14-day case-fatality in a preliminary analysis, and was therefore omitted from the logistic regression model. The second multilevel logistic regression model was used to adjust for differences between hospitals in age, sex, aneurysm treatment modality, and severity and comorbidity markers. In this second model 
Table I. Characteristics of the 8525 aSAH patients per hospital volume grouping

\begin{tabular}{|c|c|c|c|}
\hline & $\begin{array}{l}\text { Group } \\
\text { I - Median } \\
\text { annual volume } \\
<4 I n=2363\end{array}$ & $\begin{array}{l}\text { Group } 2 \text { - Median } \\
\text { annual volume } \\
41-70 \\
n=3563\end{array}$ & $\begin{array}{l}\text { Group } \\
3-\text { Median } \\
\text { annual volume } \\
>70 n=2599\end{array}$ \\
\hline & $n(\%)$ & $n(\%)$ & $n(\%)$ \\
\hline \multicolumn{4}{|l|}{ Age (years) } \\
\hline$\leq 50$ years & $896(38 \%)$ & 1416 (40\%) & $94 \mathrm{I}(36 \%)$ \\
\hline \multicolumn{4}{|l|}{$\operatorname{Sex}(\%)$} \\
\hline Female sex & $1614(68 \%)$ & $2402(67 \%)$ & $1746(67 \%)$ \\
\hline \multicolumn{4}{|l|}{ Type of aneurysm treatment (\%) } \\
\hline Coiling & $1370(58 \%)$ & $2129(60 \%)$ & $1170(45 \%)$ \\
\hline Clipping & $993(42 \%)$ & 1434 (40\%) & $1429(55 \%)$ \\
\hline \multicolumn{4}{|l|}{ Comorbidity (\%) } \\
\hline Cardiac arrhythmias & $355(15 \%)$ & $504(14 \%)$ & $254(10 \%)$ \\
\hline Fluid and electrolyte disorders & $939(40 \%)$ & $1070(30 \%)$ & $602(23 \%)$ \\
\hline Valvular disease & $38(2 \%)$ & $62(2 \%)$ & $37(1 \%)$ \\
\hline Rheumatoid arthritis collagen vascular disease & $36(2 \%)$ & $57(2 \%)$ & $45(2 \%)$ \\
\hline Peptic ulcer disease excluding bleeding & II (I\%) & $7(1 \%)$ & $2(1 \%)$ \\
\hline Peripheral vascular disorders & $186(8 \%)$ & $304(9 \%)$ & $148(6 \%)$ \\
\hline Chronic pulmonary disease & $188(8 \%)$ & $336(10 \%)$ & $213(8 \%)$ \\
\hline Solid tumor without metastasis & $21(1 \%)$ & $22(1 \%)$ & $15(1 \%)$ \\
\hline Diabetes complicated & $28(1 \%)$ & $34(1 \%)$ & $13(1 \%)$ \\
\hline Metastatic cancer & $6(1 \%)$ & $6(1 \%)$ & $3(1 \%)$ \\
\hline Aids HIV & $3(1 \%)$ & $0(0 \%)$ & $3(1 \%)$ \\
\hline Lymphoma & $\mathrm{I}(\mathrm{l} \%)$ & $5(1 \%)$ & $3(1 \%)$ \\
\hline Obesity & $63(3 \%)$ & $|4|(4 \%)$ & $59(2 \%)$ \\
\hline Alcohol abuse & $82(4 \%)$ & $157(4 \%)$ & 77 (3\%) \\
\hline Drug abuse & $69(3 \%)$ & $|3|(4 \%)$ & $120(5 \%)$ \\
\hline Congestive heart failure & $124(5 \%)$ & $150(4 \%)$ & $67(3 \%)$ \\
\hline Diabetes uncomplicated & II 15 (5\%) & $245(7 \%)$ & 170 (7\%) \\
\hline Paralysis & $348(15 \%)$ & $382(11 \%)$ & $159(6 \%)$ \\
\hline Renal failure & $43(2 \%)$ & $54(2 \%)$ & $39(2 \%)$ \\
\hline Hypertension uncomplicated & $1011(43 \%)$ & 1448 (4I\%) & $922(36 \%)$ \\
\hline
\end{tabular}


Table I. Continued

\begin{tabular}{|c|c|c|c|}
\hline & $\begin{array}{l}\text { Group } \\
\text { I - Median } \\
\text { annual volume } \\
<4 \text { I } n=2363\end{array}$ & $\begin{array}{l}\text { Group } 2 \text { - Median } \\
\text { annual volume } \\
41-70 \\
n=3563\end{array}$ & $\begin{array}{l}\text { Group } \\
3-\text { Median } \\
\text { annual volume } \\
>70 n=2599\end{array}$ \\
\hline Hypertension complicated & $31(1 \%)$ & $35(1 \%)$ & $32(1 \%)$ \\
\hline Coagulopathy & $101(4 \%)$ & $91(3 \%)$ & $48(2 \%)$ \\
\hline Length of stay, days & 17 & 16 & 15 \\
\hline Crude I4-day case-fatality (\%) & $246(10 \%)$ & $250(7 \%)$ & $140(5 \%)$ \\
\hline
\end{tabular}

14-day case-fatality was again used as the outcome, and median annual volume, age, sex, count of co-morbidities, and aneurysm treatment modality as fixed effects variables. Low hospital volume was used as reference in all comparisons. To account for the variation of admission criteria and thereby variation in disease severity between hospitals, hospital was used as a random effects variable. In addition, we ran the multilevel model in the subgroups of clipped and coiled patients separately. The study protocol was submitted to and approved by the Scientific and Research Committee of Dr Foster Global Comparators before data analyses were performed.

\section{Role of the funding source}

The sponsors had no role in study design, data collection, data analysis, data interpretation, or writing of the report. The authors had full access to all data in the study and had final responsibility for the decision to submit for publication.

The authors declare that all supporting data are available within the article.

\section{Results}

During the study period, 8525 aSAH patients had aneurysm occlusion by means of clipping or coiling. The characteristics of the patients are shown in Table 1.

Median annual volume of aSAH patients treated per year ranged from 19 to 136 . Crude case-fatality in relation to median annual hospital aSAH case-volumes are- given in Figure 1. Crude 14-day case-fatality for hospitals with low case-volume was $10.4 \%$ (95\% CI $9.2-11.7 \%)$, for hospitals with intermediate casevolume $7.0 \%$ (95\% CI $6.2-7.9 \%)$ and for hospitals with high case-volume $5.4 \% \quad(95 \%$ CI $4.6-6.3 \%)$ (Figure 2).

OR for case-fatality within 14 days was $0.65(95 \%$ CI $0.54-0.78)$ for the intermediate hospital casevolume and 0.51 (95\% CI 0.41-0.64) for the high hospital case-volume, using low volume as a reference. Other variables associated with 14-day case-fatality were treatment with coiling (OR 1.20; 95\% CI 1.20, 1.01-1.42), cardiac arrhythmias (OR 1.48; 95\% CI 1.18-1.85), diabetes (OR 1.47; 95\% CI 1.10-1.97), renal failure (OR 2.69; 95\% CI 1.33-5.41), uncomplicated hypertension (OR 1.38; 95\% CI 1.15-1.64), coagulopathy (OR 2.20; 95\% CI 1.52-3.18), and fluid and electrolyte disorders (OR $0.81,95 \%$ CI $0.67-0.99$ ).

In the second multilevel logistic regression model, with hospital as random effects variable, OR for death at 14 days was 0.63 (95\% CI $0.47-0.86)$ for intermediate hospital case-volume and 0.50 (95\% CI 0.33 $0.74)$ for high hospital case-volume (Table 2). In the subgroup of patients with clipped aneurysms, the OR for 14-day case-fatality was $0.46(95 \%$ CI $0.30-0.71)$ for intermediate hospital case-volume and 0.42 (95\% CI $0.25-0.72$ ) for high hospital case-volume. In the subgroup of patients with coiled aneurysms, OR was 0.77 (95\% CI 0.55-1.07) for intermediate hospital casevolume and 0.56 (95\% CI 0.36-0.87) for high hospital case-volume.

\section{Discussion}

We showed in an extensive, international administrative dataset of large, tertiary care centers that higher hospital case-volume is associated with lower 14-day case-fatality after clipping or coiling of the ruptured aneurysm in aSAH patients, regardless of treatment modality. Furthermore, our results suggest that this association exists beyond the previously used definitions of high-volume hospital.

Since widely acknowledged definitions for high and low case-volume centers do not exist, thresholds for high and low volume centers differed between various studies and recommendations. The current guideline of the American Heart and Stroke Association for subarachnoid hemorrhage treatment states that "low-volume hospitals ( $<10$ aSAH cases per year) should consider early transfer of patients with aSAH to high-volume centers (eg, >35 aSAH patients 
Figure I. Crude 14-day case-fatality rates after aSAH and aneurysm occlusion plotted against hospital case-volumes for the included 22 hospitals shown with logarithmic fit lines: All patients (top panel), only patients treated with clipping (middle panel), and only patients treated with coiling (bottom panel). Hospital case-volume grouping indicated by colors.
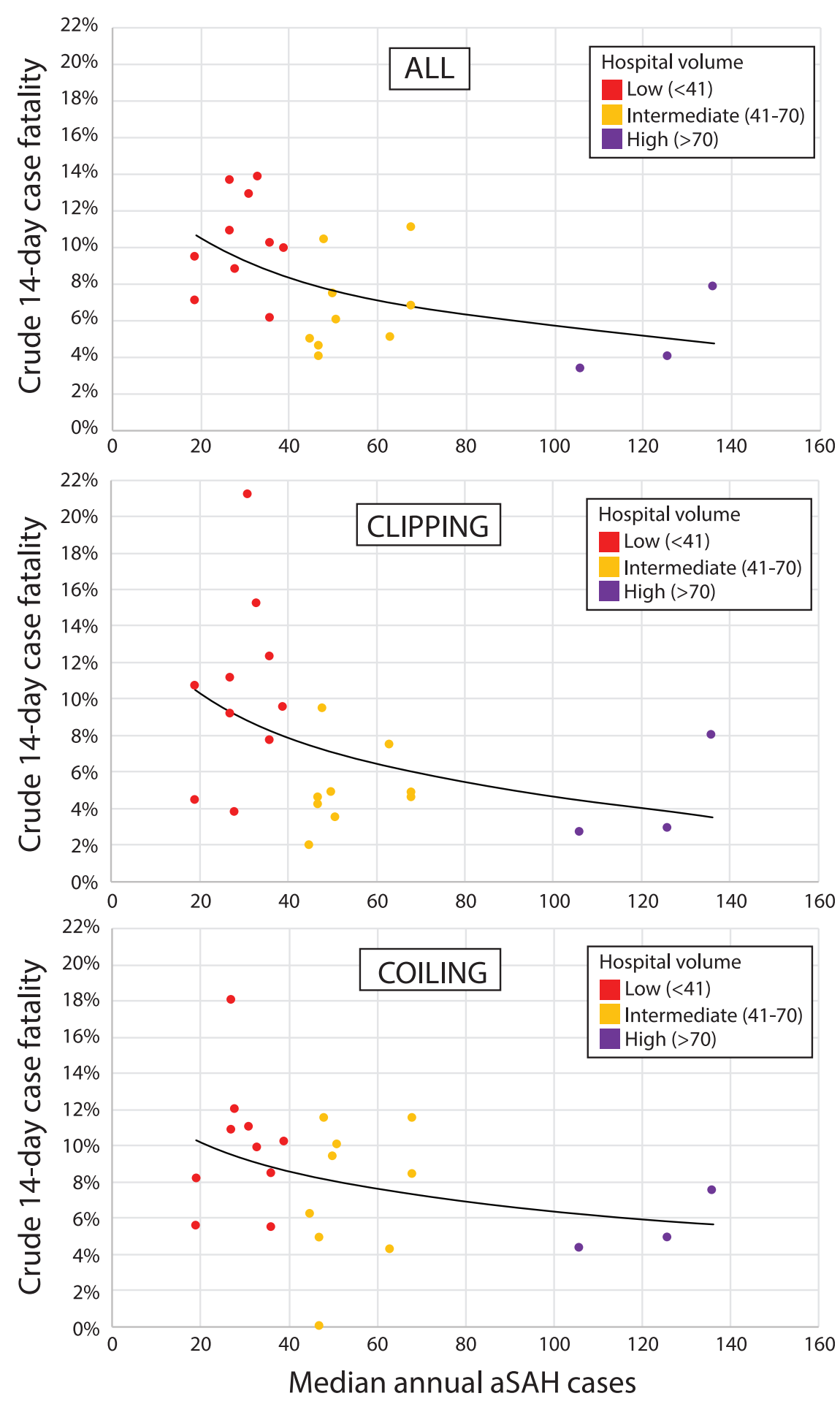
Figure 2. Crude 14-day case-fatality and $95 \%$ confidence intervals in hospital case-volume groups.

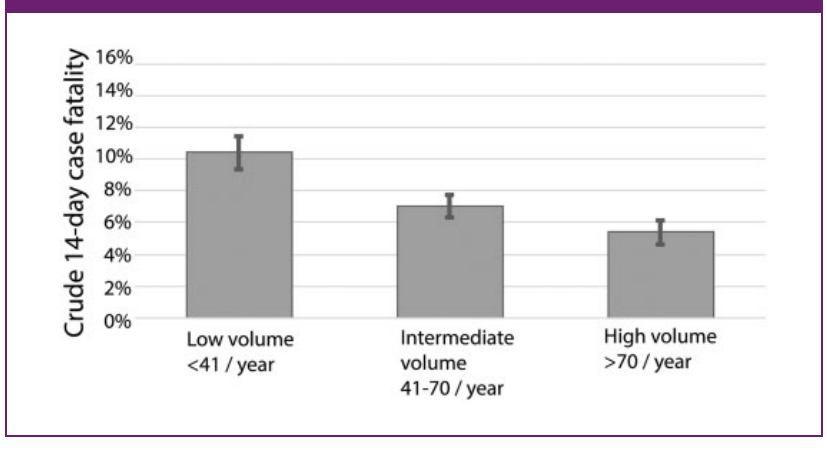

per year) with experienced cerebrovascular surgeons, endovascular specialists, and multidisciplinary neurointensive care services". 19 The US Joint Commission requires at least 20 aSAH patients to be treated yearly to qualify for being an advanced stroke center. These limits of 20 or 35 patients per year for high centers fall within our category of low centers and our data show that transfer of patients to centers treating at least 70 patients per year is associated with better outcome. This conclusion is supported by the fact that hospitals that contributed data to our study are all large academic or teaching facilities. This rather homogeneous set of hospitals contributes to the internal validity of our data. Our results indicate that even amongst this selected group of hospitals, a clear volume-case-fatality correlation exists in treatment of aSAH. There was no statistically significant difference in case-fatality between intermediate and high-volume centers, but the trend was towards lower case-fatality in highvolume centers compared to intermediate volume centers. This result remains to be validated future studies. In resource-poor areas, intermediate case-volume may be an adequate initial target for achievement.

After publication of the International Subarachnoid Aneurysm Trial (ISAT), the availability of endovascular treatment of intracranial aneurysms has increased and is now widely available also in smaller centers. ${ }^{20}$ A recent study from the United States showed that patients who were treated in high-volume centers were more likely to receive endovascular treatment of the ruptured aneurysm. ${ }^{6}$ This could reflect the better availability of interventional neuroradiology services in high-volume hospitals. However, this was not the case in our study. In the units with highest case-volumes, a higher proportion of aSAH patients was treated with clipping. The case-fatality after aSAH was lower in high-volume units regardless of treatment modality, which may indicate that treatment modalities are selected more appropriately in these hospitals. In this study, we adjusted for treatment modality in multivariable models and ran the statistical model in treatment modality subgroups. However, we were not able to adjust for level of experience of the treating physician. In Scandinavia, treatment of ruptured aneurysms is exclusively performed in academic hospitals with geographical, mutually exclusive catchment areas and good availability of both vascular neurosurgery and interventional neuroradiology services, in contrast to the United States where neurosurgical services are more likely to be offered by general neurosurgeons in low-volume centers. This may be a factor that diluted the association between hospital volume and outcome after aSAH in the Scandinavian international study. ${ }^{13}$

Only one previous study on hospital case-volume and outcome reported clinical data describing the aSAH severity on admission. ${ }^{14}$ In this series of 7578 aSAH patients with surgically occluded ruptured aneurysms from 369 centers in Japan, no association was found between hospital case-volume and clinical outcome at discharge. However, the analysis in that study was restricted to surgically treated patients and no multivariable analysis was performed. In our study, the effect of hospital volume was, in fact, slightly stronger in the subgroup of patients treated by clipping.

Our study has some limitations. First, the study is a nonrandomized retrospective study. Second, our results rely on administrative data and we did not have data on clinical status on admission. Instead, we used comorbidity scores derived from administrative data to attempt to control for disease severity. Even though the dataset is large and international, it is susceptible to errors in coding as all administrative registries, namely by mistakenly coded diagnoses as well as interventions. Since we rely on administrative dataset, we could not verify the diagnoses of aSAH. However, given the rarity of traumatic and infectious aneurysms and the fact that all included patients had undergone aneurysm treatment with either coiling or clipping, the bias introduced by wrong diagnoses is likely to be small. Third, we included only patients treated with coiling or clipping, while aSAH patients without aneurysm treatment were excluded. Thus, our results should be interpreted as the correlation of hospital casevolumes and surgical or endovascular treatment outcome, rather that aSAH outcome as a whole. Fourth, we did not have data of functional outcome or longterm mortality. A strong point of our study is the large, international dataset derived from tertiary care hospitals with high number of aSAH patients, which resulted in high numbers of patients also in the category with low case-volume. As a consequence, the variation within the categories is less extreme than in previous studies (with highest case-volume categories including hospitals with 20 to $>200$ /year). Furthermore, we only included patients who received treatment for their 
Table 2. ORs with $95 \% \mathrm{Cl}$ for 14-day case-fatality after aSAH from the multilevel multivariable logistic regression model for all patients and the clipping and coiling subgroups

\begin{tabular}{|c|c|c|c|}
\hline Variable & All $\quad(n=8525)$ & Clipping $\quad(n=3856)$ & Coiling $\quad(n=4669)$ \\
\hline \multicolumn{4}{|c|}{ Median annual SAH case-volume } \\
\hline$<4$ I & Ref & Ref & Ref \\
\hline $4 I-70$ & $0.63(0.47-0.85)$ & $0.46(0.30-0.7 \mathrm{I})$ & $0.77(0.55-1.07)$ \\
\hline$>70$ & $0.50(0.33-0.74)$ & $0.42(0.25-0.72)$ & $0.56(0.36-0.87)$ \\
\hline Age $\leq 50$ & Ref & Ref & Ref \\
\hline Age $>50$ & $1.20(1.00-1.42)$ & I.I5 (0.88-I.20) & $1.23(0.98-1.55)$ \\
\hline Male sex & Ref & Ref & Ref \\
\hline Female sex & $0.96(0.81-I .14)$ & $0.92(0.70-1.20)$ & $0.99(0.79-1.24)$ \\
\hline Comorbidity index & $1.13(1.07-1.20)$ & $1.15(1.05-1.25)$ & $1.13(1.04-1.22)$ \\
\hline Clipping of aneurysm & Ref & & \\
\hline Coiling of aneurysm & $1.19(1.00-1.41)$ & & \\
\hline
\end{tabular}

Ref: reference.

Hospital was included as random effect. In test for significance between model with and without random effect $p<0.000$.

ruptured aneurysm and thus all traumatic, nonaneurysmal, and other causes of SAH have been excluded.

\section{Conclusion}

Even in this international dataset of large tertiary care hospitals, we found that treatment of aSAH in hospitals with high and intermediate case-volumes is associated with lower in-hospital case-fatality than treatment in hospitals with lower case-volumes, regardless of treatment modality. Our data suggest that currently recommended thresholds for high-volume centers in guidelines are far too low and that treatment should be concentrated to centers that treat at least 70 aSAH patients per year. Further studies are needed to investigate whether the reduced case-fatality in high-volume centers is related to better availability of specialized interventional neuroradiology, neurosurgical or neurointensive care services, timing of treatment, or other aspects of the management of these patients.

\section{Declaration of conflicting interests}

The author(s) declared no potential conflicts of interest with respect to the research, authorship, and/or publication of this article.

\section{Funding}

The author(s) received no financial support for the research, authorship, and/or publication of this article.

\section{ORCID iD}

Antti Lindgren (D) http://orcid.org/0000-0002-1264-7667

Sarah Burt (D) http://orcid.org/0000-0003-3556-0764

Mervyn DI Vergouwen (ID http://orcid.org/0000-0002-68231628

\section{References}

1. Finks J, Osborne N and Birkmeyer JD. Trends in hospital volume and operative mortality for high-risk surgery. $N$ Engl J Med 2011; 364: 2128-2137.

2. Saposnik G, Baibergenova A, O’Donnell M, et al. Hospital volume and stroke outcome: Does it matter? Neurology 2007; 69: 1142-1151.

3. Solomon RA, Mayer SA and Tarmey JJ. Relationship between the volume of craniotomies for cerebral aneurysm performed at New York state hospitals and in-hospital mortality. Stroke 1996; 27: 13-17.

4. Gonda DD, Khalessi AA, McCutcheon BA, et al. Longterm follow-up of unruptured intracranial aneurysms repaired in California: Clinical article. J Neurosurg 2014; 120: 1349-1357.

5. Pandey AS, Gemmete JJ, Wilson TJ, et al. High subarachnoid hemorrhage patient volume associated with lower mortality and better outcomes. Neurosurgery 2015; 77: 462-470.

6. Rush B, Romano K, Ashkanani M, Mcdermid RC and Celi LA. Impact of hospital case-volume on subarachnoid hemorrhage outcomes: A nationwide analysis adjusting for hemorrhage severity. J Crit Care 2017; 37: 240-243. 
7. McNeill L, English SW, Borg N, Matta BF and Menon DK. Effects of institutional caseload of subarachnoid hemorrhage on mortality: A secondary analysis of administrative data. Stroke 2013; 44: 647-652.

8. Berman MF, Solomon RA, Mayer SA, Johnston SC and Yung PP. Impact of hospital-related factors on outcome after treatment of cerebral aneurysms. Stroke 2003; 34: 2200-2205.

9. Leake CB, Brinjikji W, Kallmes DF and Cloft HJ. Increasing treatment of ruptured cerebral aneurysms at high-volume centers in the United States. J Neurosurg 2011; 115: 1179-1183.

10. Andaluz $N$ and Zuccarello $M$. Recent trends in the treatment of cerebral aneurysms: Analysis of a nationwide inpatient database. $J$ Neurosurg 2008; 108: 1163-1169.

11. Bardach NS, Zhao S, Gress DR, Lawton MT and Johnston SC. Association between subarachnoid hemorrhage outcomes and number of cases treated at California hospitals. Stroke 2002; 33: 1851-1856.

12. Cross DT, Tirschwell DL, Clark MA, et al. Mortality rates after subarachnoid hemorrhage: Variations according to hospital case volume in 18 states. J Neurosurg 2003; 99: 810-817.

13. Lindekleiv H, Mathiesen EB, Forde O, Wilsgaard T and Ingebrigtsen $\mathrm{T}$. Hospital volume and 1-year mortality after treatment of intracranial aneurysms: A study based on patient registries in Scandinavia. J Neurosurg 2015; 123: 631-637.

14. Hattori N, Katayama Y and Abe T; Japan Neurosurgical Society. Case volume does not correlate with outcome after cerebral aneurysm clipping: A nationwide study in Japan. Neurol Med Chir 2007; 47: 95-101.

15. Chang TR, Kowalski RG, Carhuapoma JR, Tamargo RJ and Naval NS. Impact of case volume on aneurysmal subarachnoid hemorrhage outcomes. J Crit Care 2015; 30: 469-472.

16. Johnston SC. Effect of endovascular services and hospital volume on cerebral aneurysm treatment outcomes. Stroke 2000; 31: 111-117.

17. The Royal College of Surgeons of England. National study of subarachnoid haemorrhage: Final report of an audit carried out in 34 neurosurgical units in the $U K$ and Ireland between 14 September 2001 to 13 September 2002. London, UK: The Royal College of Surgeons of England, 2006.

18. Bottle A, Middleton S, Kalkman CJ, Livingston EH and Aylin P. Global comparators project: International comparison of hospital outcomes using administrative data. Health Serv Res 2013; 48: 2081-2100.

19. Connolly ES Jr, Rabinstein AA, Carhuapoma JR, et al. Guidelines for the management of aneurysmal subarachnoid hemorrhage: A guideline for healthcare professionals from the American Heart Association/American Stroke Association. Stroke 2012; 43: 1711-1737.

20. Molyneux A, Kerr R, Stratton I, et al. International Subarachnoid Aneurysm Trial (ISAT) of neurosurgical clipping versus endovascular coiling in 2143 patients with ruptured intracranial aneurysms: A randomised trial. Lancet 2002; 360: 1267-1274. 